\title{
Volume holographic grating-based continuously tunable optical filter
}

\author{
Frank Havermeyer \\ Wenhai Liu \\ Christophe Moser \\ Ondax, Incorporated \\ 850 East Duarte Road \\ Monrovia, California 91016 \\ E-mail: havermeyer@ondax.com \\ Demetri Psaltis, FELLOW SPIE \\ California Institute of Technology \\ Department of Electrical Engineering \\ Pasadena, California 91125 \\ Gregory J. Steckman \\ Ondax, Incorporated \\ 850 East Duarte Road \\ Monrovia, California 91016
}

\begin{abstract}
We propose and demonstrate a widely tunable optical filter, realized by angle tuning a volume holographic grating. The volume holographic grating selectively drops a narrow portion of the signal bandwidth into a fiber while passing through the rest of the signals. The demonstrated 1510 - to $1590-\mathrm{nm}$ tuning range covers the entire erbiumdoped fiber amplifier (EDFA) $\mathrm{C}$ band, with small bandwidth variation and low insertion loss $(<1 \mathrm{~dB})$. Group delay, polarization-dependent loss, and polarization mode dispersion are measured and investigated for optimizing the filter characteristics. () 2004 Society of Photo-Optical Instrumentation Engineers. [DOI: 10.1117/1.1773775]
\end{abstract}

Subject terms: tunable optical filter; volume holographic grating; wavelengthdivision multiplexing.

Paper VHOE-S06 received Nov. 30, 2003; revised manuscript received Mar. 23, 2004; accepted for publication Apr. 9, 2004.

\section{Introduction}

Wavelength-division multiplexing (WDM) has extended fiber optics from simple point-to-point links to flexible largecapacity networks capable of dynamic wavelength routing for efficient capacity management. Such dynamic optical networks require reconfigurable optical components to control the optical channels for optimum network performance. ${ }^{1}$ One key component in WDM networks is the tunable optical filter, which provides the flexibility in wavelength control and routing. Its essential requirements include wide tuning range, low insertion loss, low chromatic dispersion, low polarization-dependent loss (PDL), low polarization mode dispersion (PMD), high stopband rejection, fast tuning speed, small size, and low cost. ${ }^{2}$

Available single-passband tunable optical filter techniques include fiber Bragg gratings (FBGs), ${ }^{3}$ thin film filters (TFTs) ${ }^{4}$ Fabry-Perot filters (FPF), ${ }^{5}$ arrayed waveguide gratings (AWGs), ${ }^{6}$ acousto-optic tunable filters (AOTFs), ${ }^{7}$ and microelectro-mechanically-actuated tunable filters. ${ }^{8}$ The FBG gives the best tunable optical filter because of its sharp spectral filtering characteristics, low insertion loss, and chromatic dispersion compensation capability with proper chirping and apodization along the grating. However, the FBG is difficult to be tuned for wide range by either thermal tuning or mechanical stress.

Volume holographic gratings (VHGs) are in many ways similar to FBGs except that the recording medium for the grating is not a single-mode fiber but a volume medium. Consequently, the incident and diffracted light are not confined to the modes of the fiber, but can be assigned to any mode that can propagate in the volume of the material. This opens up a new set of possibilities for the design of useful devices, and allows additional flexibility such as removal of the circulator, which is essential for FBGs. VHGs have been previously used as optical filters ${ }^{9}$ and demultiplexers. ${ }^{10,11}$ We investigate and demonstrate a VHG- based continuously tunable filter. It achieves a wide tuning range $(1510$ to $1590 \mathrm{~nm})$ and low insertion loss $(<1 \mathrm{~dB})$.

\section{Volume Holographic Grating Angle Tuning}

Figure 1 shows a schematic representation of the continuously angle-tunable volume holographic filter. The Bragg wavelength of a holographic grating is determined by: ${ }^{12}$

$\lambda_{b}=2 n \Lambda \cos \theta$,

where $\lambda_{b}$ is the Bragg phase matching wavelength, $n$ is the refractive index of the material, $\Lambda$ is the holographic grating period, and $\theta$ is the angle of incidence inside the holographic material. By changing the incident angle $\theta$, the Bragg wavelength can be tuned continuously from $2 n \Lambda$ down to $2 \Lambda \sqrt{\left(n^{2}-1\right)}$. For practical application, the tuning range can easily cover the entire $\mathrm{C}$ band, limited mainly by the higher insertion loss at larger angle $\theta$, which is caused by: 1. the sharp angle selectivity of the VHG at a large angle $\theta$ compared with the angle deviation of a finite aperture Gaussian beam, and 2. the coupling efficiency between a large aperture Gaussian beam and fiber due to the geometry limits. The critical requirement to make this idea a practical tunable filter device is to collect the drop signal into a fiber during the angle tuning without an expensive tracking mechanism or feedback control system. This is achieved with a self-reflector architecture that recombines the reflective holographic grating with a wideband infrared (IR) mirror, as shown in Fig. 1.

A retroreflector consists of two mirrors with a fixed angle $\alpha$ between the mirror surfaces. And the angle between the input and reflected signal becomes $\pi-2 \alpha$. For a normal retroreflector, as shown in Fig. 1(a), the IR mirrors are arranged as orthogonal to each other with $\alpha=\pi / 2$, and the reflected signal beam is always inverse to the input direction and fixed spatially when rotating the mirror struc- 


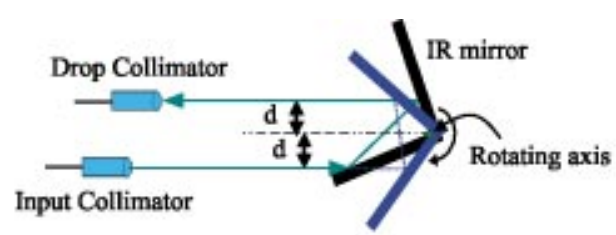

(a)

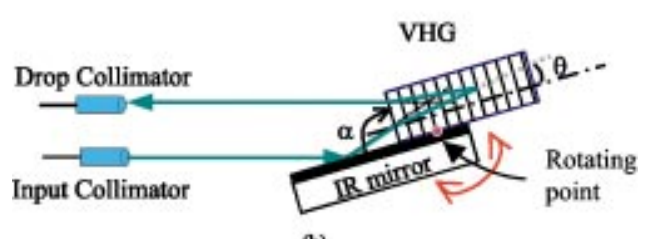

(b)

Fig. 1 (a) A normal retroreflector with two perpendicular mirrors. The reflected beam is fixed when the retroreflector is rotated around the mirror joint. (b) Replacing one mirror by a VHG. The continuously tunable wavelength of this optical filter is determined by the VHG incident angle $\theta$.

ture around the crossing point of the mirror surfaces. This also applies to the structure in Fig. 1(b), where one mirror is replaced by a VHG. At the Bragg wavelength, this VHG works as a reflective mirror with an effective depth into the material. When rotating the mirror/grating structure around the crossing point of the mirror and grating surfaces at the effective reflection depth, the drop signal beam is fixed spatially while the wavelength is tuned by the angle $\theta$.

With the addition of a temperature sensor and suitable control system, the device can be made athermal ${ }^{13}$ by compensating the angle $\theta$ to offset temperature-induced variations of the grating period $\Lambda$ in response to ambient temperature variation.

\section{Insertion Loss, Beam Size, and Experimental Demonstration}

Figure 2 shows an experimental demonstration of continuous tuning of such a device across the $\mathrm{C}$ band. The tuning range is only determined by the geometry limit of the device and the filter performance. The tunable device consists of two parallel fiber collimators with beam width $500 \mu \mathrm{m}$ as input and drop, an IR mirror, and a holographic grating of fixed angle $\alpha \sim 90 \mathrm{deg}$, as shown in Fig. 1. A tunable laser is coupled into the input fiber collimator and the diffracted signal is collected into the drop fiber collimator. The holographic grating is recorded inside a photosensitive glass with period $535 \mathrm{~nm}$, which corresponds to a Bragg wavelength $1591.5 \mathrm{~nm}$ at normal incident angle. The angle $\theta$ is tuned between 11 and 17 deg to cover the $\mathrm{C}$ band 1560 to $1528 \mathrm{~nm}$, as shown in Fig. 2(a).

Figure 2(b) shows all the overlapped filter shapes over the whole tuning range and they are similar with limited deformation. However, the insertion loss (IL) increases from 2.2 to $4 \mathrm{~dB}$ while tuning across the $\mathrm{C}$ band in Fig. 2(a). This increase of IL is due to the finite aperture of the input signal. For an input Gaussian beam with a finite beam size $w$, there is a spatial angle deviation $\sim \lambda / w$ from a perfect plane wave. However, the angle selectivity of a reflective grating $\Delta \theta=\lambda /(2 n \sin \theta L)$ improves as the tuning incident angle $\theta$ increases, where $L$ is the effective length of

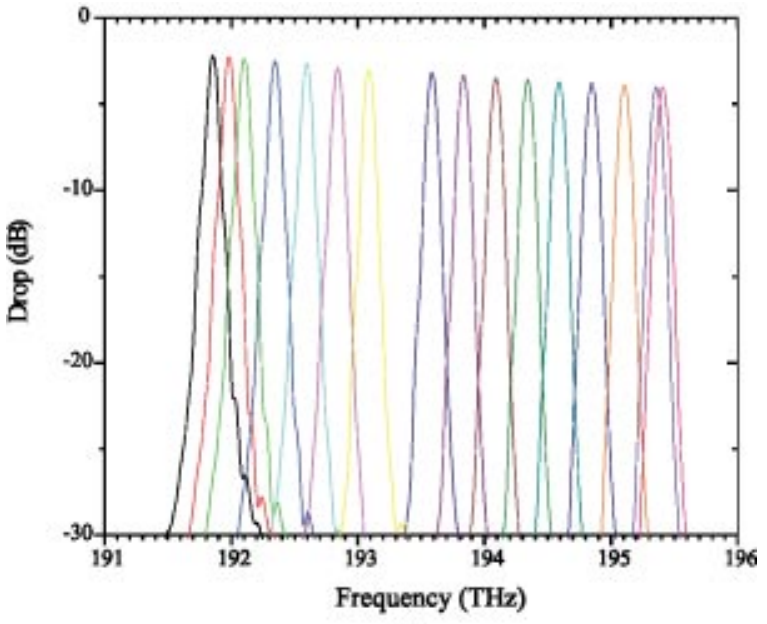

(a)

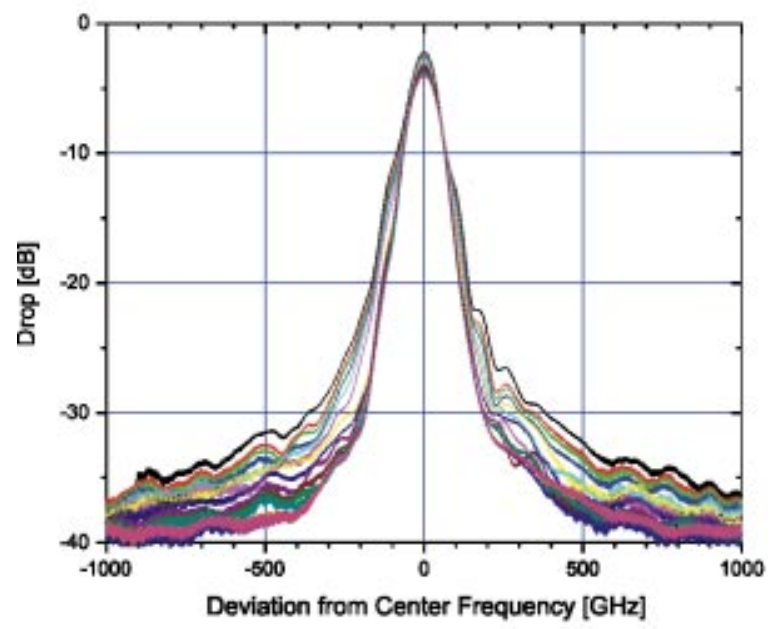

(b)

Fig. 2 (a) Continuous tuning filters over C-band with fiber collimators of $0.5-\mathrm{mm}$-diam beam size; (b) overlap of all the filter shapes with limited variation over the whole tuning range.

the grating. When $\Delta \theta$ is comparable with or smaller than the Gaussian angular deviation, part of the Gaussian beam will not be Bragg matched at the same time as the central components.

By simulating the Bragg diffraction of different Gaussian beam sizes from the holographic grating, Fig. 3 shows that the IL of a $500-\mu \mathrm{m}$-diam input beam increases $1.5 \mathrm{~dB}$ along the tuning angle 10 to $18 \mathrm{deg}$. To decrease the IL variation $<0.5 \mathrm{~dB}$ over the $\mathrm{C}$ band, the collimated beam size needs to be $>1.5 \mathrm{~mm}$ in diam. Figure 4 shows the experimental tuning filters with similar devices in Fig. 1 with collimators of $2.5-\mathrm{mm}$ beam size. The tuning range covers 1510 to $1590 \mathrm{~nm}$, and the IL variation is smaller than $0.7 \mathrm{~dB}$, as shown in Fig. 4(b). The larger than expected IL variation is random instead of systematically going down as the tuning angle increases, which could be contributed from other measurement noise such as the stability of the angular tuning and the extreme angular coupling sensitivity of the large beam collimator. The nonsymmetric filter shape, as shown in Fig. 4(b), is possibly caused by the nonuniform refractive index along the grating due to the 


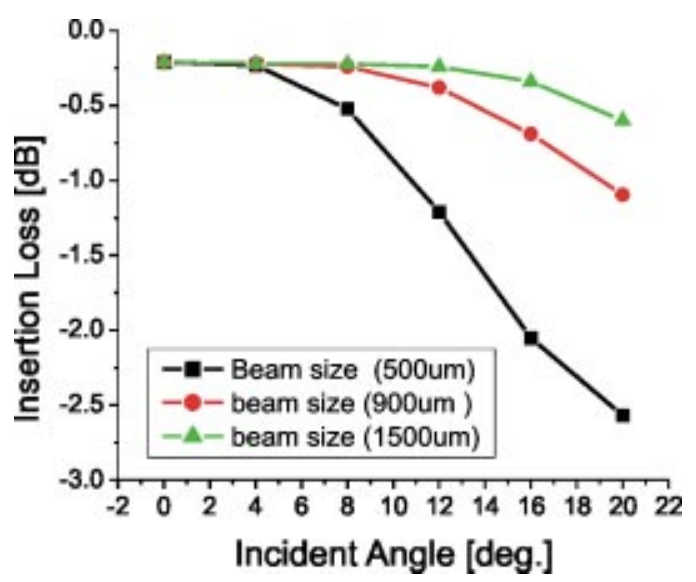

Fig. 3 Theoretical simulation on the insertion loss over the tuning angle for various Gaussian beam diameters.

nonuniform apodized exposure in the holographic material in our experiments, similar to the induced refractive index variation in FBG. Figure 5 shows the filter bandwidth measured at both -0.5 and $-3 \mathrm{~dB}$ from the maximum as the

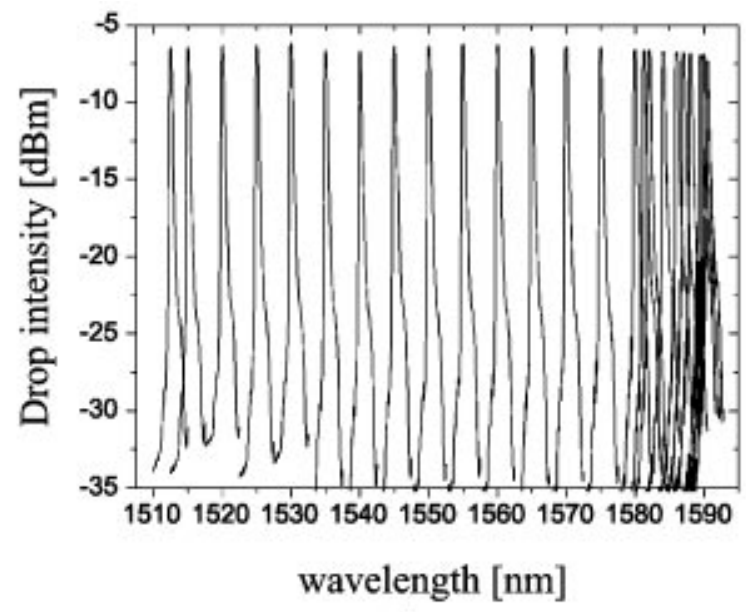

(a)

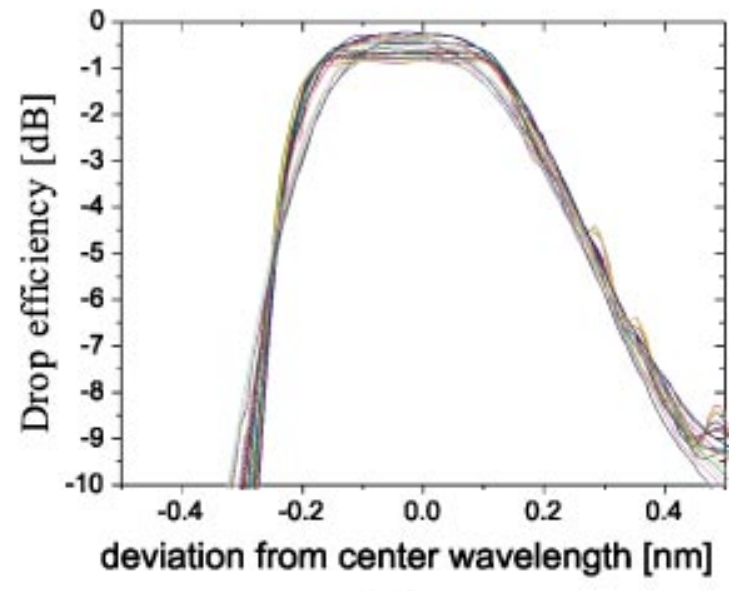

(b)

Fig. 4 (a) Continuous tuning filters over 1510 to $1590 \mathrm{~nm}$ with fiber collimators of 2.5-mm-diam beam size; (b) overlap of all the filter shapes with IL $<1 \mathrm{~dB}$ and limited variation over the whole tuning range.

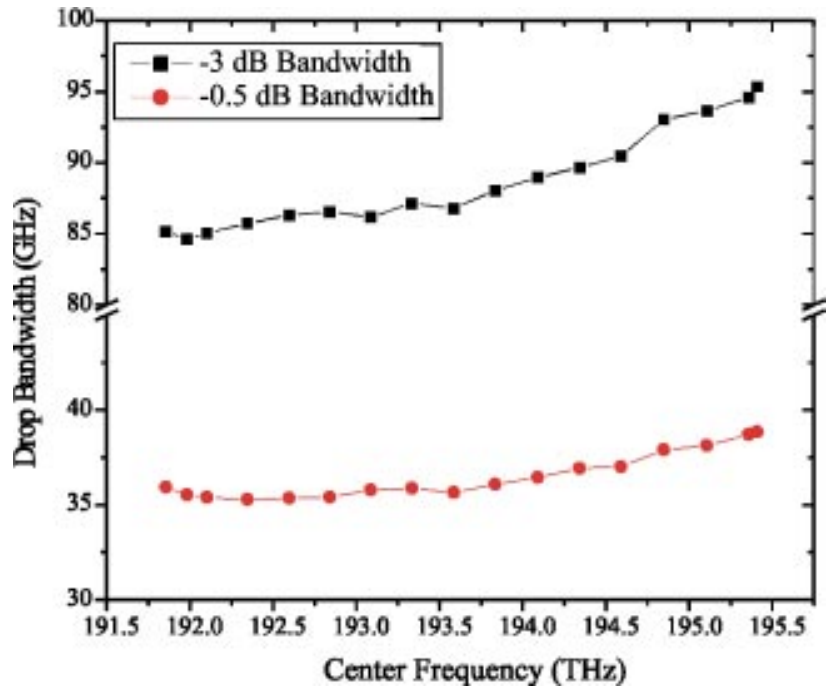

Fig. 5 Filter bandwidth while tuning over the C-band.

angle tuning across the $\mathrm{C}$ band. Less than $10 \%$ variation in bandwidth is seen over the entire tuning range.

\section{Group Delay, PDL, PMD Measurements, and Discussion}

Figure 6 measures the group delay of the tunable filter at the center of the $\mathrm{C}$ band. The group delay ripple is smaller than 5 ps within the $-3-\mathrm{dB}$ bandwidth. The bandwidth, isolation, and other performance characteristics of the filter can be tailored during fabrication to suit the application by changing the grating strength, apodization, and chirping, ${ }^{14}$ in a manner similar to the FBG.

However, unlike the FBG, the VHG is a free-space device and the input beam at a nonzero incident angle sees different effective grating strength for polarization in or out of the incident plane of the grating. This could cause high PDL and PMD in the volume holographic filter. We measured the PDL and PMD of the tunable filter at three different frequencies across the tuning range, as shown in Fig. 7. Within the tuning angle of 11 to $17 \mathrm{deg}$, PDL is smaller than $0.4 \mathrm{~dB}$ and PMD is smaller than 0.4 ps. Both PMD

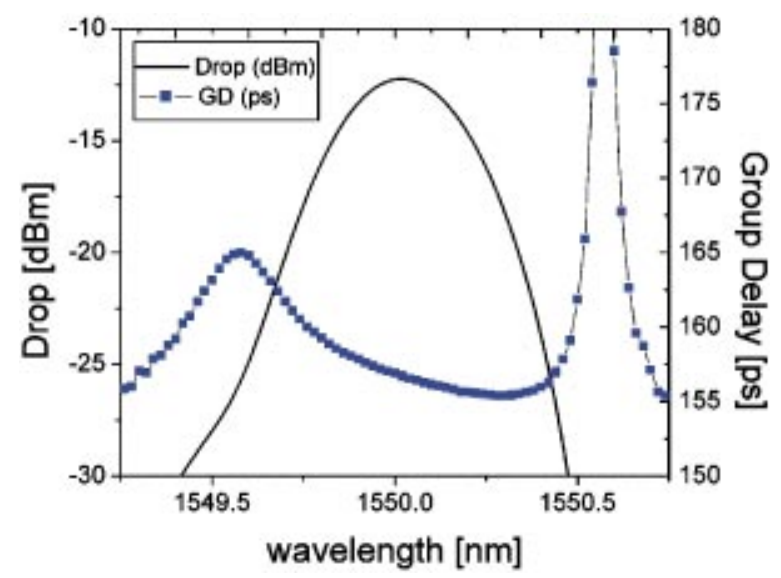

Fig. 6 Group delay measurement of the tunable filter at the center of C-band. 


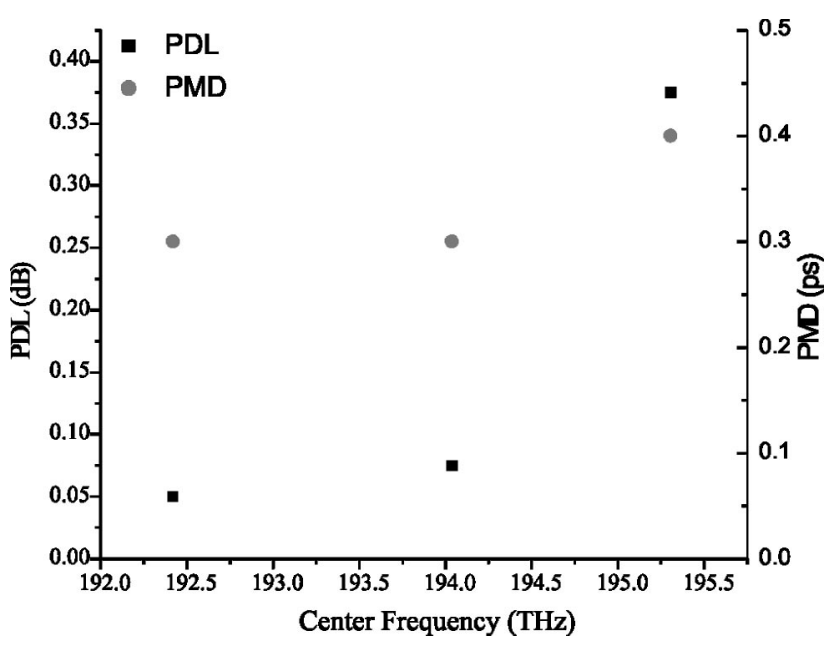

Fig. 7 Polarization dependent loss and polarization mode dispersion.

and PDL increase as the filter is tuned to higher frequencies due to the increase in the incident beam angle.

There are various methods used in optical networks to improve the PDL and PMD. ${ }^{15}$ One potential solution to minimize both PDL and PMD further is utilizing a multipass architecture, in which the beam is diffracted by the same or a different grating a second time after undergoing $90 \mathrm{deg}$ of polarization rotation. Multiple passes through a single grating can also be used to generate filters with small frequency spacing and high stopband isolation. One such approach is to replace the drop collimator in Fig. 1 with a Faraday rotation mirror (FRM). The device is constructed as previously described, but the first diffracted drop beam is instead reflected by a FRM, which rotates the polarization of the light as well as reflects it back along the original path. The reflected beam then makes a second pass through the VHG and is diffracted backward along the same path as the original input light. Since the second pass is made with light whose polarization is rotated with respect to that of the first pass, polarization-dependent effects such as PMD and PDL are reduced. However, a circulator is needed to separate the output from the input, as the normal FBG.

There are also alternative double-pass configurations achieved without using a circulator. One architecture is to use a retroreflector to reflect the drop beam backward to the grating, along a path transversely shifted from the drop beam. This beam will then diffract a second time by the VHG, reflect off the IR mirror, and be coupled into a second collimator that is parallel to the first input collimator. To compensate for PMD and PDL effects, a half-waveplate can be placed in the beam path to exchange the in/out of plane polarization components before making a second pass through the VHG.

\section{Conclusion}

Volume holographic grating maintains the sharp filter performance of a FBG while providing the addition freedom of manipulating the signal in free space. With proper design of an optical architecture, we demonstrate a simple singleparameter tunable optical filter over 1510 to $1590 \mathrm{~nm}$ and low insertion loss $<1 \mathrm{~dB}$. By combining the mature apodization/chirping FBG technique and the flexible architecture, volume holographic gratings can lead to the construction of more sophisticated continuous tunable filters, such as low PDL, PMD, and dispersion filters, or dispersion compensation.

\section{References}

1. T. E. Stern and K. Bala, Multiwavelength Optical Networks: a Layered Approach, Addision-Wesley, Reading, MA (1999).

2. D. Sadot and E. Boimovich, "Tunable optical filters for dense WDM networks," IEEE Commun. Mag. 38, 382-384 (1995).

3. A. Iocco, H. G. Limberger, R. P. Salathe, L. A. Everall, K. E. Chisholm, J. A. R. Williams, and I. Bennion, "Bragg grating fast tunable filter for wavelength division multiplexing," J. Lightwave Technol. 17, 1217-1221 (1999).

4. M. Lequine, R. Parmentier, F. Lemarchand, and C. Amra, "Toward tunable thin-film filters for wavelength division multiplexing applications," Appl. Opt. 41(16), 3277-3284 (2002).

5. J. Stone and L. W. Stulz, "Pigtailed high finesse tunable FP interferometer with large, medium and small FSR," Electron. Lett. 23, 781783 (1987)

6. T. Watanabe, Y. Inoue, K. Kaneko, N. Ooba, and T. Kurihara, "Polymeric arrayed-waveguide grating multiplexer with wide tuning range," Electron. Lett. 33(18), 1547-1548 (1997).

7. J. Jackel et al., "Acousto-optic tunable filters (AOTF's) for multiwavelength optical cross-connects: crosstalk considerations," J. Lightwave Technol. 14, 1056-1066 (1996).

8. E. Vail et al., "GaAs micromachined widely tunable Fabry-Perot filters," Electron. Lett. 31(3), 228-229 (1995).

9. V. Leyva, G. A. Rakuljic, and B. O'Conner, "Narrow bandwidth volume holographic optical filter operating at the $\mathrm{Kr}$ transition at 1547.82 nm," Appl. Phys. Lett. 65, 1079-1081 (1994).

10. S. Beer and K. Buse, "Wavelength demultiplexing with volume phase holograms in photorefractive lithium niobate," Appl. Phys. B: Laser Opt. 66, 339-345 (1998).

11. C. Moser, F. Havermeyer, W. Liu, G. Steckman, K. Buse, and D. Psaltis, "Volume Bragg grating devices," OFC 2003 Tech. Digest, pp. 644-645

12. H. Kogelnik, "Coupled wave theory for thick hologram gratings," Bell Syst. Tech. J. 84, 2909-2947 (1969).

13. H. Hsieh, G. Panotopoulos, M. Liger, Y. Tai, and D. Psaltis, "Athermal holographic filters," IEEE Photonics Technol. Lett. 16(1), 177179 (2004).

14. S. Han, B. A. Yu, S. Chung, H. Kim, J. Paek, and B. Lee, "Filter characteristics of a chirped volume holographic grating," Opt. Lett. 29(1), 107-109 (2004)

15. G. P. Agrawal, Fiber-Optic Communication Systems, 3rd Ed., pp. 236-237, Wiley, New York (2002)

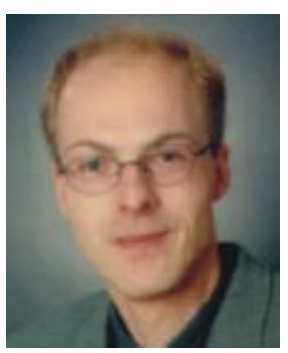

Frank Havermeyer received his diploma and $\mathrm{PhD}$ degree in physics from the University of Osnabrueck (Germany) in 1996 and 2000, respectively. After two postdoctoral positions in Germany at the GKSS Research Center and the University of Bonn he joined the start-up company Ondax (USA) in 2001. He is currently working on the design and optimization of the production process of volume phase gratings for DWDM and general wavelength filter applications.

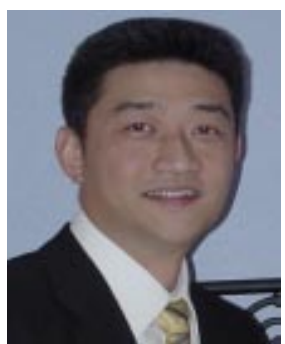

Wenhai Liu received his BS from the Tsinghua University (China) in 1992, MS in physics from the University of Chicago in 1996, and $\mathrm{PhD}$ in electrical engineering from the California Institute of Technology in 2001. He has been working on product research and development at Ondax, Inc. since 2001. His research interests are holographic technology and their applications in telecommunication and optical information processing. 


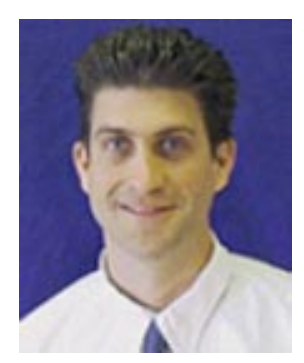

Christophe Moser is co-founder and CEO of Ondax. Prior to founding Ondax, Dr. Moser held management positions in the optical measurement system division of TESA-Brown \& Sharpe in Switzerland, and at Holoplex, an optical data storage start-up in Pasadena, California. During his thesis at Caltech, he developed devices and processes that are at the core of Ondax's technology. He received his PhD in electrical engineering from Caltech.

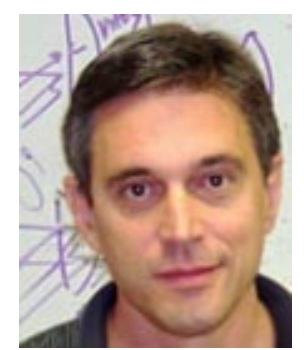

Demetri Psaltis is the Thomas G. Myers Professor of Electrical Engineering at Caltech in Pasadena, California. He was educated at Carnegie-Mellon University where he received a BS degree in electrical engineering and economics in 1974, a master's in 1975, and a PhD in electrical engineering in 1977. In 1980, he joined the faculty at the California Institute of Technology, Pasadena, California, and he served as executive officer for the Computation and Neural Systems Department from 1992 to 1996. From 1996 until 1999 he was the director of the National Science Foundation research center on Neuromorphic Systems Engineering at Caltech. $\mathrm{He}$ is currently director of the Center for Optofluidic Integration at Caltech. His research interests are in optical information processing, holography, optical networks, imaging, optical memories, and optical devices. He has authored or co-authored more than 300 publications in these areas. Dr. Psaltis is a fellow of the Optical Society of America and SPIE. He received the International Commission of Optics Prize and the Humboldt Award. He is a chairman and cofounder of Ondax.

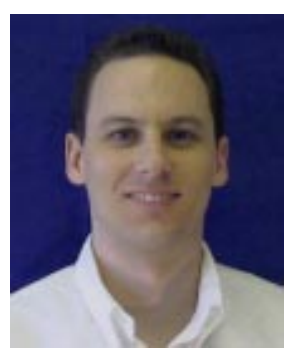

Gregory J. Steckman received his BS from the University of California, San Diego, in 1995 and his MS and PhD from the California Institute of Technology in 1997 and 2000 , respectively, all in electrical engineering. He is currently the director of engineering at Ondax, Inc., responsible for the research and design of new products. Dr. Steckman has authored or co-authored numerous publications on the subject of volume holography. 